\title{
Profile variation of bla genes among non-lactose fermenting Gram- negative bacilli between clinical and environmental isolates of Dr. Soetomo Hospital, Surabaya, Indonesia
}

\author{
PRISTIAWAN NAVY ENDRAPUTRA ${ }^{1}$, KUNTAMAN KUNTAMAN ${ }^{1,2, \vartheta}$, EDDY BAGUS WASITO ${ }^{1,2}$, \\ TOSHIRO SHIRAKAWA ${ }^{3,4,5}$, DADIK RAHARJO ${ }^{6}$, WAHYU SETYARINI ${ }^{6}$ \\ ${ }^{1}$ Department of Clinical Microbiology, Faculty of Medicine, Universitas Airlangga. Jl. Mayjen Prof. Dr. Moestopo 47, Surabaya 60132, East Java, \\ Indonesia. Tel./fax.: +62-31-5022472, ^email: kuntaman@fk.unair.ac.id \\ ${ }^{2}$ Dr. Soetomo Hospital. Jl. Mayjen Prof. Dr. Moestopo No. 6-8, Surabaya 60286, East Java, Indonesia \\ ${ }^{3}$ Division of Translational Research for Biologics, Department of Internal Related, Graduate School of Medicine, Kobe University. 7-5-1 Kusunoki-cho, \\ Chuo-ku, Kobe, 650-0017, Japan \\ ${ }^{4}$ Department of Urology, Graduate School of Medicine, Kobe University. 7-5-1 Kusunoki-cho, Chuo-ku, Kobe, 650-0017, Japan \\ ${ }^{5}$ Division of Advanced Medical Science, Graduate School of Science, Technology and Innovation, Kobe University. 7-5-1 Kusunoki-cho, Chuo-ku, \\ Kobe, 650-0017, Japan \\ ${ }^{6}$ Institute of Tropical Disease, Universitas Airlangga. Jl. Mulyorejo, Surabaya 60115, East Java, Indonesia
}

Manuscript received: 6 October 2021. Revision accepted: 25 October 2021.

\begin{abstract}
Endraputra PN, Kuntaman K, Wasito EB, Shirakawa T, Raharjo D, Setyarini W. 2021. Profile variation of bla genes among non-lactose fermenting Gram negative bacilli between clinical and environmental isolates of Dr. Soetomo Hospital, Surabaya, Indonesia. Biodiversitas 22: 5047-5054. Carbapenem-resistant non-fermenter Gram-negative bacilli are notorious opportunistic pathogens in hospitalized patients and hospital environments. This study explored the carbapenemase gene among non-fermenter Gramnegative bacilli from hospital wastewater and clinical isolates in Dr. Soetomo Hospital, Surabaya, Indonesia. All samples were screened on MacConkey agar with meropenem $2 \mu \mathrm{g} / \mathrm{ml}$ and gene detected by Multiplex PCR. All samples were screened on MacConkey agar with meropenem $2 \mu \mathrm{g} / \mathrm{ml}$ and gene detected by Multiplex PCR. A total of 121 isolates consisted of 76 clinical (41 carbapenem-resistant Acinetobacter baumannii and 35 carbapenem-resistant Pseudomonas aeruginosa), 45 environmental isolates (6 carbapenem-resistant Pseudomonas aeruginosa and 32 carbapenem-resistant Pseudomonas spp.), and 7 screening samples (all CRPAs). Clinical isolates carbapenemase genes were identified, blaoXA-23-like 21 (28\%), blaoXA-24-like 30 (39\%), blaNDM-1 1 (1\%), and blaIMP-1 6 (8\%) while environmental isolates were blaoXA-23-like 5 (13\%), blaoXA-24-like 4 (11\%), blaoXA-48-like 2 (5\%), blaNDM-1 13 (34\%), blavim 12 (32\%), and bla IMP-1 $4(11 \%)$. Rectal swab screening specimens presented bla oXA-23-like $3(43 \%)$, bla OXA-24-like $3(43 \%)$, and bla $a_{N D M-1} 1(14 \%)$. The carbapenemase gene pattern was different between clinical and environmental isolates. The blaOXA-23-like and blaOXA-24-like were most prevalent among in both clinical and wastewater, while blaVIM was mostly in wastewater. The presence of carbapenem-resistant nonfermenter Gram-negative bacilli carrying carbapenemase genes in hospital effluents indicated that the community river was seeded with an antimicrobial resistance gene.
\end{abstract}

Keywords: Acinetobacter baumannii, carbapenemase gene, OXA, Pseudomonas aeruginosa, wastewater

Abbreviations: CRAB: Carbapenem resistant Acinetobacter baumannii, CRPA: Carbapenem resistant Pseudomonas aeruginosa, CROs: Carbapenem resistant organisms, MDRO: Multi-drug resistant organism, ARGs: Antimicrobial resistant genes, MGEs: Mobile genetic elements, CR-NFGNB: Carbapenem-resistant non-fermenter Gram negative bacilli

\section{INTRODUCTION}

Multi-drug resistant Acinetobacter baumannii and Pseudomonas aeruginosa are responsible for nosocomial infections worldwide (Kateete et al. 2016), leading to higher mortality, morbidity, longer hospital stays, and financial problems (Motbainor et al. 2020). In Southeast Asia, carbapenem-resistant Acinetobacter baumannii (CRAB) and carbapenem-resistant Pseudomonas aeruginosa (CRPA) are commonly reported causative agents for nosocomial infections (Suwantarat and Carroll 2016), part of a rising global trend in CRAB and CRPA (Li et al. 2018; Du et al. 2019). In Indonesia, the prevalence of CRAB was higher, $50.5 \%$, compared to $21.9 \%$ of CRPA in ICU (Karuniawati et al. 2013).
Carbapenems have been considered the most powerful drug in treating multi-drug resistant Gram-negative bacteria (Aruhomukama et al. 2019). Consequently, the increased use of carbapenem led to the global rise of carbapenemaseproducing Acinetobacter baumannii (Du et al. 2019; Kalal et al. 2020). This enzyme is the major mechanism for carbapenem resistance in Acinetobacter baumannii and is divided into Ambler class A, B, and D based on amino acid sequence (Hsu et al. 2017; Beigverdi et al. 2019; Bush and Bradford 2019). The most common mechanism for CRAB is the production of a carbapenemase enzyme, predominantly oxacillinase (OXA). To date, OXA-23 still dominates the OXA group (Beigverdi et al. 2019). The presence of MBLs such as NDM, IMP, SIM, VIM has been reported in Acinetobacter baumannii (Abouelfetouh et al. 
2019), with IMP being the most common, followed by VIM and NDM (Beigverdi et al. 2019). Hence, this rising phenomenon resulted in a critical situation in antibiotic availability against the MDR phenotype of Acinetobacter baumannii infections (Lukovic et al. 2020).

Pseudomonas aeruginosa presents with multiple antibiotic resistance through intrinsic, acquired, or adaptive mechanisms (Botelho et al. 2019), including its ability to acquire B-lactamases (serine-type, MBLs, or OXAs). Even though distribution varies geographically, VIM, IMP, and NDM have been identified worldwide (Moradali et al. 2017).

The high number of antibiotic-resistant bacteria-harboring antimicrobial-resistant genes (ARGs) in the environment has been a health issue (Barancheshme and Munir 2018). Hospital environments are pooled for carbapenem-resistant organisms (CROs). Studies demonstrate that hospital wastewater hosts a variety of ARGs and resistant enteric bacteria (Marathe et al. 2019). The interplay between mobile genetic elements (MGEs), resistant bacteria, and commensals which serve as vectors for ARGs may spread into the bacterial community through horizontal gene transfer (Asfaw 2018; Marathe et al. 2019; Fouz et al. 2020). It has been hypothesized that ARGs in humans likely originate from the environment (Fouz et al. 2020).

Detection of antibiotic-resistant bacteria or ARGs by vulture or molecular based technique. As the culture-based method can recover living bacteria carrying ARGs, the molecular method may identify any ARGs from dead or alive bacteria and thus expand the probability of observing potential transfer (Rizzo et al. 2013). As previously reported, the presence of antibiotic-resistant bacteria in wastewater spotlights the possible seeding of these bacteria into the environment (Cahill et al. 2019). While significantly reducing the number of bacteria, the chlorination process potentially increases antibioticresistant bacteria (Rizzo et al. 2013). The study by Huang et al. (2013) demonstrated tetracycline-resistant Escherichia coli was resistant to high dose chlorination compared to susceptible strains. Following treatment, hospital wastewater will be emitted into the environment. However, it has been reported that hospital water treatment is ineffective in eliminating multi-drug resistant bacteria and or the ARGs and, therefore, significantly impacts the spread of carbapenemase producers (Dziri et al. 2020). The existence of multi-drug Acinetobacter baumannii outside the hospital may be a public concern since it may serve as a vector of resistance within the community environment (Anane et al. 2019).

This study aimed to analyze carbapenemase genes in carbapenem-resistant non-fermenter Gram-negative bacilli (CR-NFGNB), particularly Acinetobacter and Pseudomonas species, among clinical and environmental isolates.

\section{MATERIALS AND METHODS}

\section{Bacterial isolates and identification}

All clinical isolates of $41 \mathrm{CRABs}$ and 35 CRPAs were collected from sputum, blood, urine, and wound specimens between mid-August to mid-November 2020 from intensive care unit, emergency department, surgical, pediatric, and medical ward excluding isolation, also urine and wound isolates from a previous study at RSUD Dr. Soetomo, Surabaya (Kuntaman et al. 2018) from those admitted to RSUD Dr. Soetomo in 2015 and 2019 respectively. These isolates were identified by BD Phoenix ${ }^{\mathrm{TM}}$ automated identification and susceptibility testing system, following the manufacturer's instructions. Carbapenem-resistant organisms were defined based on their resistance towards either meropenem or imipenem according to CLSI 2020 breakpoint for Acinetobacter baumannii and Pseudomonas aeruginosa (Table 1).

Environmental samples were collected twice a week from late August to mid-September 2020 by collecting 500 $\mathrm{mL}$ samples using pre-sterilized laboratory bottles from wastewater adjacent towards (surgery, internal medicine, pediatric, obstetrics and gynecology (ObGy), and intensive care unit (ICU)), hospital effluent, and inanimate objects in the ICU. Rectal swabs from ICU patients were also collected as MDRO screening. $45 \mathrm{~mL}$ of wastewater sample was processed by 10 minutes centrifugation at $3000 \mathrm{xg}$ at $4^{\circ} \mathrm{C}$ and subsequently inoculating sediment onto in-house MacConkey agar by pouring $1 \mu \mathrm{L}$ of $2 \mu \mathrm{g} / \mathrm{mL}$ meropenem stock solution into every $\mathrm{mL}$ of molten MacConkey agar (final concentration of $2 \mu \mathrm{g} / \mathrm{mL}$ meropenem) as previously described (Zhang et al. 2020a) with modification. Rectal swabs were inoculated in BHI broth and initially incubated for 4 hours. One loop of BHI suspension was streaked on in-house MacConkey agar supplemented with $2 \mu \mathrm{g} / \mathrm{mL}$ meropenem and incubated $37^{\circ} \mathrm{C}$ overnight. The ethical committee granted ethical approval in health research of Dr. Soetomo Hospital, Surabaya, through the following number 0065/LOE/301.4.2/ VII/2020. Informed consent was obtained from patients in ICU prior to specimen collection.

Following incubation, visible growth of non-fermenter colonies was taken for catalase and oxidase tests. DNA was extracted by boiling and submitted to multiplex PCR to identify Acinetobacter baumannii and Acinetobacter spp., with a total of $20 \mu \mathrm{L}$ for each PCR reaction $(5 \mu \mathrm{L}$ DNA template, $0.67 \mu \mathrm{L}$ each primer, $10 \mu \mathrm{L}$ GoTaq Master Mix Promega), starting at $94^{\circ} \mathrm{C}$ for 2 minutes for initial denaturation, then 25 cycles of denaturation at $94^{\circ} \mathrm{C}$ for 1 minute, annealing at $60^{\circ} \mathrm{C}$ for 30 seconds, extension at $72^{\circ} \mathrm{C}$ for 1 minute, and a final extension at $72^{\circ} \mathrm{C}$ for 10 minutes (Higgins et al. 2007). Pseudomonas aeruginosa and Pseudomonas spp. were identified by multiplex PCR with a total of $20 \mu \mathrm{L}$ for each PCR reaction $(5 \mu \mathrm{L}$ DNA template, $0.5 \mu \mathrm{L}$ each primer, $10 \mu \mathrm{L}$ GoTaq Master Mix Promega). Multiplex PCR was started with an initial denaturation at $94^{\circ} \mathrm{C}$ for 5 minutes, then 30 cycles of denaturation at $94^{\circ} \mathrm{C}$ for 1 minute, annealing at $55^{\circ} \mathrm{C}$ for 1 minute, extension at $72^{\circ} \mathrm{C}$ for 1 minute, and a final extension at $72^{\circ} \mathrm{C}$ for 10 minutes (Gholami et al. 2016). The primers for each detection are described in Table 2. 
Table 1. Phenotypic identification of CRAB and CRPA from clinical isolates

\begin{tabular}{lllcc}
\hline $\begin{array}{c}\text { Specimen } \\
\text { type }\end{array}$ & Ward & MDRO & Meropenem & Imipenem \\
\hline Sputum & Medical & CRAB & $\mathrm{R}$ & $\mathrm{R}$ \\
Sputum & Surgical & CRAB & $\mathrm{R}$ & $\mathrm{R}$ \\
Sputum & Medical & CRAB & $\mathrm{R}$ & $\mathrm{R}$ \\
Sputum & Medical & CRAB & $\mathrm{R}$ & $\mathrm{R}$ \\
Sputum & Medical & CRAB & $\mathrm{R}$ & $\mathrm{R}$ \\
Wound & Surgical & CRAB & $\mathrm{R}$ & $\mathrm{R}$ \\
Sputum & Medical & CRAB & $\mathrm{R}$ & $\mathrm{R}$ \\
Blood & Medical & CRAB & $\mathrm{R}$ & $\mathrm{R}$ \\
Sputum & Medical & CRAB & $\mathrm{R}$ & $\mathrm{R}$ \\
Sputum & Pediatric & CRAB & $\mathrm{R}$ & $\mathrm{R}$ \\
Blood & Surgical & CRAB & $\mathrm{R}$ & $\mathrm{R}$ \\
Blood & Surgical & CRAB & $\mathrm{R}$ & $\mathrm{R}$ \\
Sputum & Pediatric & CRAB & $\mathrm{R}$ & $\mathrm{R}$ \\
Wound & Medical & CRAB & $\mathrm{R}$ & $\mathrm{R}$ \\
Sputum & Pediatric & CRAB & $\mathrm{R}$ & $\mathrm{R}$ \\
Sputum & Medical & CRPA & $\mathrm{R}$ & $\mathrm{R}$ \\
Sputum & Pediatric & CRAB & $\mathrm{R}$ & $\mathrm{R}$ \\
Blood & Pediatric & CRAB & $\mathrm{R}$ & $\mathrm{R}$ \\
Wound & Pediatric & CRAB & $\mathrm{R}$ & $\mathrm{R}$ \\
Sputum & Medical & CRAB & $\mathrm{R}$ & $\mathrm{R}$ \\
Sputum & Medical & CRAB & $\mathrm{R}$ & $\mathrm{R}$ \\
Sputum & ED & CRAB & $\mathrm{R}$ & $\mathrm{R}$ \\
Sputum & Medical & CRPA & $\mathrm{R}$ & $\mathrm{R}$ \\
Wound & Medical & CRPA & $\mathrm{R}$ & $\mathrm{R}$ \\
Wound & Medical & CRAB & $\mathrm{R}$ & $\mathrm{R}$ \\
Blood & Pediatric & CRAB & $\mathrm{R}$ & $\mathrm{R}$ \\
Sputum & Medical & CRAB & $\mathrm{R}$ & $\mathrm{R}$ \\
Sputum & Medical & CRAB & $\mathrm{R}$ & $\mathrm{R}$ \\
Blood & Surgical & CRAB & $\mathrm{R}$ & $\mathrm{R}$ \\
Blood & Surgical & CRAB & $\mathrm{R}$ & $\mathrm{R}$ \\
Wound & Surgical & CRAB & $\mathrm{R}$ & $\mathrm{R}$ \\
Sputum & Pediatric & CRPA & $\mathrm{I}$ & $\mathrm{R}$ \\
Sputum & Medical & CRAB & $\mathrm{R}$ & $\mathrm{R}$ \\
Sputum & Medical & CRAB & $\mathrm{R}$ & $\mathrm{R}$ \\
Sputum & Surgical & CRAB & $\mathrm{R}$ & $\mathrm{R}$ \\
\hline Sputum & Medical & CRAB & $\mathrm{R}$ & $\mathrm{R}$ \\
\hline & Medical & CRAB & $\mathrm{R}$ & $\mathrm{R}$ \\
\hline
\end{tabular}

\begin{tabular}{|c|c|c|c|c|}
\hline Wound & Surgical & CRPA & $\mathrm{R}$ & $\mathrm{R}$ \\
\hline Urine & Surgical & CRAB & $\mathrm{R}$ & $\mathrm{R}$ \\
\hline Urine & Medical & CRAB & $\mathrm{R}$ & $\mathrm{R}$ \\
\hline Wound & Medical & CRPA & $\mathrm{R}$ & $\mathrm{R}$ \\
\hline Blood & Medical & CRAB & $\mathrm{R}$ & $\mathrm{R}$ \\
\hline Sputum & Medical & CRAB & $\mathrm{R}$ & $\mathrm{R}$ \\
\hline Sputum & Medical & CRAB & $\mathrm{R}$ & $\mathrm{R}$ \\
\hline Blood & Medical & CRAB & $\mathrm{R}$ & $\mathrm{R}$ \\
\hline Wound & Surgical & CRAB & $\mathrm{R}$ & $\mathrm{R}$ \\
\hline Sputum & Medical & CRAB & $\mathrm{R}$ & $\mathrm{R}$ \\
\hline Sputum & Medical & CRPA & $\mathrm{R}$ & $\mathrm{R}$ \\
\hline Sputum & Medical & CRPA & $\mathrm{R}$ & $\mathrm{R}$ \\
\hline Blood & Medical & CRPA & $\mathrm{R}$ & $\mathrm{R}$ \\
\hline Sputum & ED & CRPA & $\mathrm{R}$ & I \\
\hline Wound & Medical & CRPA & $\mathrm{R}$ & $\mathrm{R}$ \\
\hline Sputum & Medical & CRPA & $\mathrm{R}$ & $\mathrm{R}$ \\
\hline Urine & Medical & CRPA & $\mathrm{R}$ & $\mathrm{R}$ \\
\hline Sputum & Pediatric & CRPA & $\mathrm{R}$ & $\mathrm{R}$ \\
\hline Wound & ED & CRPA & $\mathrm{R}$ & $\mathrm{R}$ \\
\hline Urine & Surgical & CRPA & $\mathrm{R}$ & $\mathrm{R}$ \\
\hline Urine & $\mathrm{ED}$ & CRPA & $\mathrm{R}$ & $\mathrm{R}$ \\
\hline Urine & Medical & CRPA & $\mathrm{R}$ & - \\
\hline Urine & Medical & CRPA & $\mathrm{R}$ & $\mathrm{R}$ \\
\hline Urine & Surgical & CRPA & $\mathrm{R}$ & $\mathrm{R}$ \\
\hline Urine & Surgical & CRPA & $\mathrm{R}$ & $\mathrm{S}$ \\
\hline Urine & Surgical & CRPA & $\mathrm{R}$ & $\mathrm{R}$ \\
\hline Urine & Medical & CRPA & S & $\mathrm{S}$ \\
\hline Urine & Pediatric & CRPA & $\mathrm{R}$ & $\mathrm{R}$ \\
\hline Urine & Medical & CRPA & $\mathrm{R}$ & $\mathrm{R}$ \\
\hline Urine & Medical & CRPA & $\mathrm{R}$ & $\mathrm{R}$ \\
\hline Urine & Surgical & CRPA & $\mathrm{R}$ & $\mathrm{R}$ \\
\hline Urine & $\mathrm{ED}$ & CRPA & $\mathrm{R}$ & $\mathrm{R}$ \\
\hline Wound & Pediatric & CRPA & $\mathrm{R}$ & $\mathrm{R}$ \\
\hline Wound & Surgical & CRPA & $\mathrm{R}$ & $\mathrm{R}$ \\
\hline Wound & Medical & CRPA & $\mathrm{R}$ & $\mathrm{R}$ \\
\hline Wound & ED & CRPA & $S$ & $\mathrm{R}$ \\
\hline Wound & Medical & CRPA & $\mathrm{R}$ & $\mathrm{R}$ \\
\hline Wound & Surgical & CRPA & I & $\mathrm{R}$ \\
\hline Wound & Medical & CRPA & $\mathrm{R}$ & $\mathrm{R}$ \\
\hline
\end{tabular}

Table 2. Primers for identification of Pseudomonas aeruginosa and Pseudomonas spp.; Acinetobacter baumannii and Acinetobacter spp.

\begin{tabular}{|c|c|c|c|c|}
\hline Genus & Target & Sequence $\left(5^{\prime}-3^{\prime}\right)$ & $\begin{array}{c}\text { Amplicon } \\
\text { (bp) }\end{array}$ & Refs \\
\hline \multirow[t]{4}{*}{ Acinetobacter } & gyrB & & & \\
\hline & $S p 2 F$ & GTT CCT GAT CCG AAA TTC TCG & $490^{*}$ & Higgins et al. (2007); \\
\hline & $S p 4 F$ & CAC GCC GTA AGA GTG CAT TA & $294 *$ & Higgins et al. (2010) \\
\hline & $\operatorname{Sp} 4 R$ & AAC GGA GCT TGT CAG GGT TA & & \\
\hline \multirow[t]{4}{*}{ Pseudomonas } & oprL & & & \\
\hline & $F$ & ATG GAA ATG CTG AAA TTC GGC & $504 * *$ & De Vos et al. (1997); \\
\hline & $\begin{array}{l}R \\
o p r I\end{array}$ & CTT CTT CAG CTC GAC GCG ACG & & Taee et al. (2014) \\
\hline & $\begin{array}{l}F \\
R\end{array}$ & $\begin{array}{l}\text { ATG AAC AAC GTT CTG AAA TTC TCT GCT } \\
\text { CTT GCG GCT GGC TTT TTC CAG }\end{array}$ & $249 * *$ & $\begin{array}{l}\text { De Vos et al. (1997; Gholami } \\
\text { et al. (2016) }\end{array}$ \\
\hline
\end{tabular}

Note: *: Acinetobacter baumannii is identified by 2 amplicons while Acinetobacter spp. is identified by only 1. **: Pseudomonas aeruginosa is identified by 2 amplicons while Pseudomonas spp. is identified by only 1 


\section{DNA extraction and bla gene detection}

DNA samples from all CRAB, CRPA and CRPs were isolated by boiling method and analyzed for carbapenemase genes in two groups of multiplex PCRs. The first group was run to detect class $\mathrm{B}$ carbapenemase genes, with a total of $20 \mu \mathrm{L}$ for each PCR reaction $(5 \mu \mathrm{L}$ DNA template, 0.3 each $\mu \mathrm{L}$ primer, $10 \mu \mathrm{L}$ GoTaq Master Mix Promega) under the following conditions: initial denaturation at $95^{\circ} \mathrm{C}$ for 5 minutes, continued by 30 cycles of denaturation at $95^{\circ} \mathrm{C}$ for 30 seconds, annealing at $58^{\circ} \mathrm{C}$ for 30 seconds, extension at $72^{\circ} \mathrm{C}$ for 30 seconds, and a final extension at $72^{\circ} \mathrm{C}$ for 5 minutes. The second group was performed to detect class $\mathrm{D}$ carbapenemase genes, with a total of $20 \mu \mathrm{L}$ each PCR reaction $(5 \mu \mathrm{L}$ DNA template, 0.25 each $\mu \mathrm{L}$ primer, $10 \mu \mathrm{L}$ GoTaq Master Mix Promega) under the following conditions: initial denaturation at $94^{\circ} \mathrm{C}$ for 5 minutes, continued by 30 cycles of denaturation at $94^{\circ} \mathrm{C}$ for 25 seconds, annealing at $52^{\circ} \mathrm{C}$ for 40 seconds, extension at $72^{\circ} \mathrm{C}$ for 50 seconds, and a final extension at $72^{\circ} \mathrm{C}$ for 6 minutes. The primers for each detection are listed in Table 3.

\section{Data analysis}

Data were analyzed by chi-square using SPSS 25.00 with $95 \%$ confidence interval and presented into tables.

\section{RESULTS AND DISCUSSION}

In total, 121 isolates consisted of 76 clinical (41 CRAB and 35 CRPA), 38 environmental isolates (6 CRPA and 32 CRPs), and 7 screening samples (all CRPAs). We identified 58 genes of 76 clinical isolates $(76.3 \%), 40$ genes of 38 environmental isolates (105.3\%), and 7 genes from 7 screening isolates $(100 \%)$ which may carry $\geq 1$ gene in a single isolate. As shown in Table 4, some non-fermenters failed to meet molecular identification and therefore were excluded.

The distribution of bla genes within the clinical specimen was dominated by sputa, with a total of 23 isolates harboring a single bla gene. Single bla gene was detected in 9 of 10 carbapenem-resistant isolates from blood specimens. We observed multiple bla genes in one isolate from urine and wound specimens. In urine specimens, 6 isolates with two bla genes and 4 isolates with single bla gene while in a wound, we found only one isolate with multiple bla genes and 8 isolates with single bla gene (Table 5).

These clinical isolates also showed the high number of bla genes recovered from medical wards among others with total of thirty-five isolates. We identified 10 blaOXA-23-like, 21 blaOXA-24-like, 1 blaNDM-1, and 3 blaIMP-1. In surgical wards, we found 4 isolates harboring two bla genes and 9 isolates with single bla gene comprised of 5 blaOXA-23-like, 9 blaOXA-24-like, dan 3 blaIMP-1. On the other hand, we only observed 6 isolates with single bla gene, blaoXA-23-like, from the pediatric ward (Table 6).

Thirty-nine genes from the environmental isolates were identified from wastewater comprised of 4 blaOXA-23-like, 4 blaOXA-24-like, 2 blaOXA-48-like, 13 blanDM-1, 12 blavIM, dan 4 blaIMP-1 and originated 12 genes from the ICU (2 blaOXA-23-like, 2 blaOXA-24-like, 1 blaOXA-48-like, 2 blaNDM-1, 4 blavim, and 1 blaImP-1), 10 from ObGy (1 blaoXA-24-like, 1 blaOXA-48-like, 5 bla $a_{N D M-1}$, and 3 bla VIM), 6 from internal medicine (2 blaoXA-23-like, 1 blanDM-1, 2 blavIM, and 1 blaIMP-1), 6 from surgery (1 blaOXA-24-like, 2 blanDM-1, 1 blavIM, and 1 blaIMP-1), 4 from hospital effluent (2 bla $a_{N D M}-1$ and 2 blavIM), and 1 (bla NDM -1 ) from the paediatric area. Total 7 isolates from rectal swabs yielded 7 genes (3 blaOXA-23-like, 3 blaOXA-24-like, and 1 bla bDM-1) and 1 gene (blaOXA-23-like) was acquired from inanimate objects in the ICU (Table 6).

The prevalence of bla OXA-23-like, bla $a_{O X A-24-l i k e}$, and bla $a_{N D M}$ ${ }_{1}$ from clinical CRAB was $21(51 \%), 17(41 \%)$, and $1(2 \%)$ respectively. CRPA results were 13 (37\%) for bla OXA-24-like and $6(17 \%)$ for bla IMP-I. $_{\text {. }}$ Gene detection in environmental CRPA revealed 6 (46\%) bla OXA-23-like, 7 (54\%) bla OXA-24-like, $1(8 \%)$ bla $_{O X A-48-l i k e,} 2(15 \%)$ bla $_{N D M-1}$, and $2(15 \%)$ bla IMP-I. $_{\text {. }}$ CRPs results were 2 (6\%) bla OXA-23-like, 1 (3\%) bla OXA-48-like, $12(38 \%)$ bla $_{N D M-1}, 12(38 \%)$ and $2(6 \%)$ for bla $a_{V I M}$ and bla IMP-1 $_{\text {IM }}$ respectively (Table 7). None of the isolates harboured bla $a_{X A-58-l i k e}$ and only two wastewater isolates were positive for blaOXA-48-like.

Table 3. Primers for detection of carbapenemase genes

\begin{tabular}{|c|c|c|c|c|}
\hline Target & & Sequence (5'-3') & $\begin{array}{c}\text { Amplicon } \\
\text { (bp) }\end{array}$ & Refs \\
\hline \multirow[t]{2}{*}{ blaoXA-23-like } & $\mathrm{F}$ & GAT CGG ATT GGA GAA CCA GA & 501 & Woodford et al. (2006) \\
\hline & $\mathrm{R}$ & ATT CTT GAC CGC ATT TCC AT & & \\
\hline \multirow[t]{2}{*}{ blaoXA-24-like } & $\mathrm{F}$ & GGT TAG TTG GCC CCC TTA AA & 246 & Woodford et al. (2006) \\
\hline & $\mathrm{R}$ & AGT TGA GCG AAA AGG GGA TT & & \\
\hline \multirow[t]{2}{*}{ blaoXA-48-like } & $\mathrm{F}$ & TTG GTG GCA TCG ATT ATC GG & 744 & Poirel et al. (2012) \\
\hline & $\mathrm{R}$ & GAG CAC TTC TTT TGT GAT GGC & & \\
\hline \multirow[t]{2}{*}{ blaoXA-58-like } & $\mathrm{F}$ & AAG TAT TGG GGC TTG TGC TG & 599 & Woodford et al. (2006) \\
\hline & $\mathrm{R}$ & CCC CTC TGC GCT CTA CAT AC & & \\
\hline \multirow[t]{2}{*}{ bla $_{N D M-1}$} & $\mathrm{~F}$ & CTG AGC ACC GCA TTA GCC & 754 & Pfeifer et al. 2011) \\
\hline & $\mathrm{R}$ & GGG CCG TAT GAG TGA TTG C & & \\
\hline \multirow[t]{2}{*}{ bla $_{V I M}$} & $\mathrm{~F}$ & TGG GCC ATT CAG CCA GAT C & 510 & Nordmann and Poirel (2002); Nishio et al. \\
\hline & $\mathrm{R}$ & ATG GTG TTT GGT CGC ATA TC & & $(2004)$ \\
\hline \multirow[t]{2}{*}{ blaImP-1 } & $\mathrm{F}$ & CTA CCG CAG CAG AGT CTT TG & 587 & Nishio et al. (2004) \\
\hline & $\mathrm{R}$ & AAC CAG TTT TGC CTT ACC AT & & \\
\hline
\end{tabular}


Table 4. Phenotypic and genotypic identification of Acinetobacter spp. and Pseudomonas spp. from environmental and MDRO screening sample

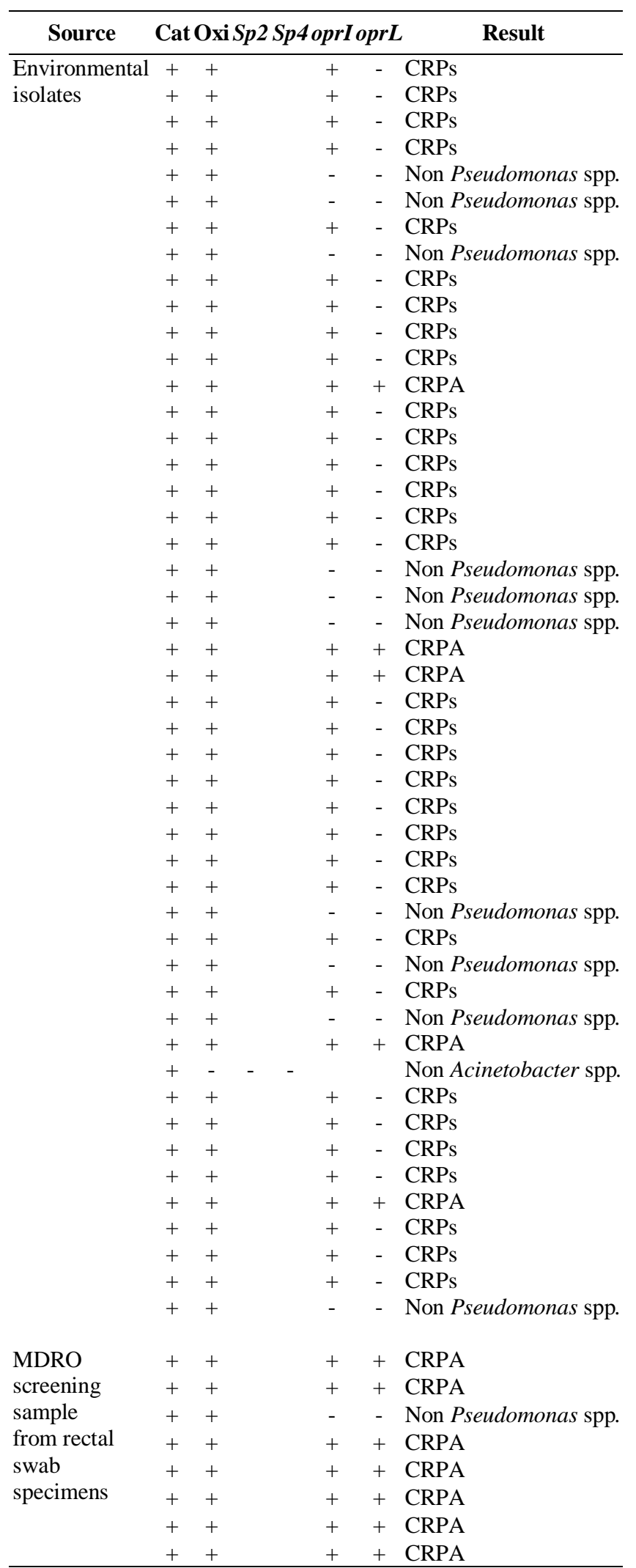

Table 5. Carbapenemase gene distribution of CRAB and CRPA based on clinical specimens

\begin{tabular}{lcccc}
\hline \multirow{2}{*}{ Specimen } & \multicolumn{4}{c}{ Gen bla } \\
\cline { 2 - 5 } & $\boldsymbol{O X} \boldsymbol{A}-23$ & $\boldsymbol{O X} \boldsymbol{A}-\mathbf{2 4}$ & $\boldsymbol{N D M}-\boldsymbol{1}$ & $\boldsymbol{I M P}-\boldsymbol{1}$ \\
\hline Blood & $6(67 \%)$ & $2(22 \%)$ & $1(11 \%)$ & $0(0 \%)$ \\
Sputum & $10(43 \%)$ & $13(57 \%)$ & $0(0 \%)$ & $0(0 \%)$ \\
Urine & $1(10 \%)$ & $9(90 \%)$ & $0(0 \%)$ & $6(60 \%)$ \\
Wound & $4(44 \%)$ & $6(67 \%)$ & $0(0 \%)$ & $0(0 \%)$ \\
\hline
\end{tabular}

\section{Discussion}

The predominance of carbapenem-resistant non-lactose fermenting Gram-negative bacilli was originated from sputum specimen compared to blood, urine, or wound. This result was consistent with the study of Cai et al. (2017) and Ssekatawa et al. (2018) which they reported the high number of CRO in respiratory tract specimens.

The prevalence of CRAB and CRPA in our hospital reached $43 \%$ and $19 \%$ in the first 6 months of 2020 (unpublished data). Clinical isolates from medical wards outnumbered others with dominant blaOXA-23- like in CRAB. This finding was also in agreement to Beigverdi et al. (2019). In clinical CRAB, bla OXA-23-like remained dominant, as found by Azimi et al. (2020), but we noticed a high number of bla $a_{\text {OXA-24-like }}$ close to bla OXA-23-like $_{\text {Tafreshi et }}$ al. (2019) explained the rise of bla $a_{O X A-24-l i k e}$ was due to increased cephalosporin use. In one isolate of CRAB, the only carbapenemase gene was $b l a_{N D M-1}$. Chatterjee et al. (2016) hypothesized that this gene was likely linked to Tn125 and not plasmid related.

In clinical CRPA, bla $a_{O X A-24-l i k e}$ outnumbered others. It is speculated that this gene might be disseminated horizontally since bla $a_{O X A-24 / 40}$ is located in either plasmid or chromosome (Vrancianu et al. 2020). Moreover, $46 \%$ of clinical CRPA did not harbor any carbapenemase genes, and resistance was due to mechanisms other than enzymes. The most identified bla genes originated from the ICU area where the use of antibiotics was excessive and the remnants might have low minimum inhibitory concentration, thus potential to induce selective pressure within surrounding wastewater, generating a variety of ARGs. In our hospital, carbapenem, for instance, was administered for patients with previous MDRO infection or critically ill patients in ICU and hence likely promoted the rise of CRO through ARG transfer beyond wastewater also excreted antibiotic which might be sub-inhibitory and possibly inducing selective pressure within surrounding wastewater.

Unexpectedly, we identified CRPs carrying carbapenemase genes from hospital effluent. Those bacteria might persist during the wastewater treatment process. This finding is supported by a study in Brazil (Miranda et al. 2015) which found Pseudomonas aeruginosa after 5 stages of hospital wastewater treatment. Wastewater treatment is less effective for removing ARGs (Zhang et al. 2020b). 
Table 6. Carbapenemase gene distribution of CRAB, CRPA, and CRPs

\begin{tabular}{|c|c|c|c|c|c|c|c|c|c|c|}
\hline & & \multirow{2}{*}{ Bacteria } & \multicolumn{8}{|c|}{ n } \\
\hline & & & $O X A-23$ & $O X A-24$ & $O X A-48$ & $O X A-58$ & NDM-1 & VIM & IMP-1 & Total \\
\hline \multirow[t]{6}{*}{ Clinical } & \multirow[t]{2}{*}{ Medical } & CRAB & 10 & 13 & - & - & 1 & - & - & 24 \\
\hline & & CRPA & - & 8 & - & - & - & - & 3 & 11 \\
\hline & \multirow[t]{2}{*}{ Pediatric } & CRAB & 6 & - & - & - & - & - & - & 6 \\
\hline & & CRPA & - & - & - & - & - & - & - & - \\
\hline & \multirow[t]{2}{*}{ Surgical } & CRAB & 5 & 4 & - & - & - & - & - & 9 \\
\hline & & CRPA & - & 5 & - & - & - & - & 3 & 8 \\
\hline \multirow[t]{14}{*}{ Environment } & \multirow[t]{2}{*}{ ICU } & CRPA & 2 & 2 & - & - & - & - & - & 4 \\
\hline & & CRPs & - & - & 1 & - & 2 & 4 & 1 & 8 \\
\hline & \multirow[t]{2}{*}{ Internal Medicine } & CRPA & - & - & - & - & - & - & 1 & 1 \\
\hline & & CRPs & 2 & - & - & - & 1 & 2 & - & 5 \\
\hline & \multirow[t]{2}{*}{ ObGy } & CRPA & - & 1 & 1 & - & 1 & - & - & 3 \\
\hline & & CRPs & - & - & - & - & 4 & 3 & - & 7 \\
\hline & \multirow[t]{2}{*}{ Pediatric } & CRPA & - & - & - & - & - & - & - & - \\
\hline & & CRPs & - & - & - & - & 1 & - & - & 1 \\
\hline & \multirow{2}{*}{ Surgical } & CRPA & - & 1 & - & - & - & - & 1 & 2 \\
\hline & & CRPs & - & - & - & - & 2 & 1 & 1 & 4 \\
\hline & \multirow[t]{2}{*}{ Hospital Effluent } & CRPA & - & - & - & - & - & - & - & - \\
\hline & & CRPs & - & - & - & - & 2 & 2 & - & 4 \\
\hline & \multirow{2}{*}{$\begin{array}{l}\text { Inanimate Objects } \\
\text { (ICU) }\end{array}$} & CRPA & 1 & - & - & - & - & - & - & 1 \\
\hline & & CRPs & - & - & - & - & - & - & - & - \\
\hline \multirow{2}{*}{\multicolumn{2}{|c|}{$\begin{array}{l}\text { MDRO Screening Sample } \\
\text { from Rectal Swab (ICU) }\end{array}$}} & CRPA & 3 & 3 & - & - & 1 & - & - & 7 \\
\hline & & CRPs & - & - & - & - & - & - & - & - \\
\hline \multicolumn{2}{|c|}{ Total } & & 29 & 37 & 2 & 0 & 15 & - & 10 & \\
\hline
\end{tabular}

Table 7. Prevalence of carbapenemase gene among nonfermenters

\begin{tabular}{lccc}
\hline \multicolumn{1}{c}{ bla Genes } & $\begin{array}{c}\text { Clinical } \\
(\mathbf{n = 7 6 )}\end{array}$ & $\begin{array}{c}\text { Environme } \\
\mathbf{n t a l} \\
(\mathbf{n = 3 8})\end{array}$ & $\begin{array}{c}\text { MDRO } \\
\text { Screening } \\
(\mathbf{n = 7})\end{array}$ \\
\hline $\begin{array}{l}\text { Overall } \text { bla genes }^{\text {a) b) }} \\
\text { Detected } \text { bla gene }^{\mathrm{b})}\end{array}$ & $51(67 \%)$ & $28(74 \%)$ & $4(57 \%)$ \\
OXA-23-like & $21(28 \%)$ & $5(13 \%)$ & $3(43 \%)$ \\
OXA-24-like & $30(39 \%)$ & $4(11 \%)$ & $3(43 \%)$ \\
OXA-48-like & - & $2(5 \%)$ & - \\
NDM-1 & $1(1 \%)$ & $13(34 \%)$ & $1(14 \%)$ \\
VIM & - & $12(32 \%)$ & - \\
IMP-1 & $6(8 \%)$ & $4(11 \%)$ & - \\
\hline
\end{tabular}

Note: ${ }^{\text {a) }}$ Isoltaes harbouring any bla genes, ${ }^{\text {b) }}$ bla genes per total isolates

The magnitude of Pseudomonas sp. in the environment, including hospital wastewater, is well understood (Spindler et al. 2012; Falodun et al. 2019). Pseudomonas sp. (28.2\%) was found five times higher than Acinetobacter sp. (5.6\%) from hospital wastewater in Singapore (Haller et al. 2018). We believe this dissemination of blaXX-24-like was derived from ARGs in the environment. Many environmental CRPs harboured $b l a_{N D M-1}$ and $b l a_{V I M}$ which might originate from Enterobacteriaceae since MBL genes are located in mobile genetic elements (Shams et al. 2018).

This present study did not identify any bla $a_{O X A-58-l i k e}$. This might be due to its susceptibility to carbapenem. Chen

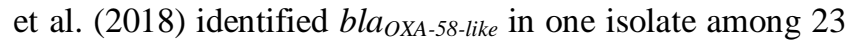
clinical CSAB with MICs of $0.5 \mu \mathrm{g} / \mathrm{mL}$ and $0.25 \mu \mathrm{g} / \mathrm{mL}$ for imipenem and meropenem respectively. Wang et al. (2018) and Ranjbar et al. (2019) successfully recovered Acinetobacter baumannii but none harboured bla OXA-58-like $_{\text {. }}$

In conclusion, we found that bla OXA-23-like and bla $a_{O X A-24-}$ like were prevalent in both clinical isolates and hospital environments while $b l a_{N D M-1}$ and $b l a_{V I M}$ were only in the hospital wastewater environment. We also identified CRNFGNB carrying carbapenemase genes in hospital water effluent, indicating that the community river was likely contaminated with ARGs and/or CROs. This alarming dissemination of a resistant bacterial community in the environment possibly constitutes a public health issue. The present study did not analyze the clonal relatedness between clinical and environmental isolates among similar bla genes. Due to this limitation, further research is needed to understand factors and other mechanisms that induce the rise of multi-drug resistant bacteria within hospital wastewater and the community water to prevent the spread of CRO.

\section{ACKNOWLEDGEMENTS}

This study was endowed by J-GRID grant from the Ministry of Education, Culture, Sports, Science, and Technology (grant number 18fm0108004h0004) and the Tahir Professorship grant of Airlangga University, Indonesia (grant number 1149/UN3/2018). Informed consent was obtained before the inclusion of rectal swabs from patients in ICU. Ethics approval was granted by Dr. Soetomo Hospital, Surabaya. 


\section{REFERENCES}

Abouelfetouh A, Torky AS, Aboulmagd E. 2019. Phenotypic and genotypic characterization of carbapenem-resistant Acinetobacter baumannii isolates from Egypt. Antimicrob Resist Infect Control 8: 185. DOI: 10.1186/s13756-019-0611-6.

Anane AY, Apalata T, Vasaikar S, Okuthe GE, Songca S. 2019. Prevalence and molecular analysis of multidrug-resistan Acinetobacter baumannii in the extra-hospital environment in Mthatha, South Africa. Braz J Infect Dis 23 (6): 371-380. DOI: 10.1016/j.bjid.2019.09.004

Aruhomukama D, Najjuka CF, Kajumbula H, Okee M, Mboowa G, Sserwadda I, Mayanja R, Joloba ML, Kateete DP. 2019. blaVIM- and blaOXA-mediated carbapenem resistance among Acinetobacter baumannii and Pseudomonas aeruginosa isolates from the Mulago hospital intensive care unit in Kampala, Uganda. BMC Infect Dis 19: 853. DOI: $10.1186 / \mathrm{s} 12879-019-4510-5$.

Asfaw T. 2018. Review on hospital wastewater as a source of emerging drug resistance pathogens. J Res Environ Sci Toxicol 7 (2): 47-52 DOI: $10.1016 \% 2 F j$.jece.2020.104812.

Azimi L, Fallah F, Karimi A, Shirdoust M, Azimi T, Sedighi I, Rahbar M, Armin S. 2020. Survey of various carbapenem-resistant mechanisms of Acinetobacter baumannii and Pseudomonas aeruginosa isolated from clinical samples in Iran. Iran J Basic Med Sci 23 (11): 13961400. DOI: 10.22038/ijbms.2020.44853.10463.

Barancheshme F, Munir M. 2018. Strategies to combat antibiotic resistance in the wastewater treatment plants. Front Microbiol 8: 2603. DOI: $10.3389 /$ fmicb.2017.02603

Beigverdi R, Sattari-Maraji A, Emaneini M, Jabalameli F. 2019. Status of carbapenem-resistant Acinetobacter baumannii harboring carbapenemase: First systematic review and meta-analysis from Iran Infect Genet Evol 73: 433-443. DOI: 10.1016/j.meegid.2019.06.008

Botelho J, Grosso F, Peixe L. 2019. Antibiotic resistance in Pseudomonas aeruginosa - mechanisms, epidemiology and evolution. Drug Resist Updat 44: 26-47. DOI: 10.1016/j.drup.2019.07.002.

Bush K, Bradford PA. 2019. Interplay between $\beta$-lactamases and new $\beta$ lactamase inhibitors. Nat Rev Microbiol 17 (5): 295-306. DOI: 10.1038/s41579-019-0159-8.

Cahill N, O'Connor L, Mahon B, Varley Á, McGrath E, Ryan P, Cormican M, Brehony C, Maiden MC, Brisse S, Morris D. 2019. Hospital effluent: A reservoir for carbapenemase-producing Enterobacterales? Sci Total Environ 672: 618-624. DOI: 10.1016/j.scitotenv.2019.03.428

Cai B, Echols R, Magee G, Ferreira JCA, Morgan G, Ariyasu M, Sawada T, Nagata TD. 2017. Prevalence of carbapenem-resistant Gramnegative infections in the United States predominated by Acinetobacter baumannii and Pseudomonas aeruginosa. Open Forum Infect Dis 4 (3): ofx176. DOI: 10.1093/ofid/ofx176.

Chatterjee S, Datta S, Roy S, Ramanan L, Saha A, Viswanathan R, Som T, Basu S. 2016. Carbapenem resistance in Acinetobacter baumannii and other Acinetobacter spp. causing neonatal sepsis: Focus on NDM1 and its linkage to ISAba125. Front Microbiol 7: 1126. DOI: 10.3389/fmicb.2016.01126

Chen Y, Yang Y, Liu L, Qiu G, Han X, Tian S, Zhao J, Chen F, Grundmann H, Li H, Sun J, Han L. 2018. High prevalence and clonal dissemination of OXA-72-producing Acinetobacter baumannii in a Chinese Hospital: A cross sectional study. BMC Infect Dis 18: 491. DOI: $10.1186 / \mathrm{s} 12879-018-3359-3$

De Vos D, Lim Jr. A, Pirnay JP, Struelens M, Vandenvelde C, Duinslaeger L, Vanderkelen A, Cornelis P. 1997. Direct detection and identification of Pseudomonas aeruginosa in clinical samples such as skin biopsy specimens and expectorations by multiplex PCR based on two outer membrane lipoprotein genes, oprI and oprL. J Clin Microbiol 35 (6): 1259-1299. DOI: $10.1128 / \mathrm{jcm} .35 .6 .1295$ 1299.1997

Du X, Xu X, Yao J, Deng K, Chen S, Shen Z, Yang L, Feng G. 2019. Predictors of mortality in patients infected with carbapenem-resistant Acinetobacter baumannii: A systematic review and meta-analysis. Am J Infect Control 47 (9): 1140-1145. DOI 10.1016/j.ajic.2019.03.003.

Dziri O, Dziri R, Ali El Salabi A, Chouchani C. 2020. Carbapenemase producing Gram-negative bacteria in Tunisia: History of thirteen years of challenge. Infect Drug Resist 13: 4177-4191. DOI: 10.2147/IDR.S259562.
Falodun OI, Akinbamiro TF, Rabiu AG. 2019. Hospital wastewater: Reservoir of antibiotic resistant Pseudomonas strains in Ibadan, Nigeria. Emer Life Sci Res 5 (1): 1-7. DOI: 10.31783/elsr.2019.5117.

Fouz N, Pangesti KNA, Yasir M, Al-Malki AL, Azhar EI, Hill-Cawthorne GA, El Ghany MA. 2020. The contribution of wastewater to the transmission of antimicrobial resistance in the environment: Implications of mass gathering settings. Trop Med Infect Dis 5 (1): 33. DOI: 10.3390/tropicalmed5010033.

Gholami A, Majidpour A, Talebi-Taher M, Boustanshenas M, Adabi M. 2016. PCR-based assay for the rapid and precise distinction of Pseudomonas aeruginosa from other Pseudomonas species recovered from burns patients. J Prev Med Hyg 57 (2): 81-85.

Haller L, Chen H, Ng C, Le TH, Koh TH, Barkham T, Sobsey M, Gin KYH. 2018. Occurrence and characteristics of extended-spectrum Blactamase- and carbapenemase-producing bacteria from hospital effluents in Singapore. Sci Total Environ 615: 1119-1125. DOI: 10.1016/j.scitotenv.2017.09.217.

Higgins PG, Wisplinghoff H, Krut O, Seifert H. 2007. A PCR-based method to differentiate between Acinetobacter baumannii and Acinetobacter genomic species 13TU. Clin Microbiol Infect 13 (12): 1199-1201. DOI: 10.1111/j.1469-0691.2007.01819.x.

Higgins PG, Lehmann M, Wisplinghoff H, Seifert H. 2010. gyrB multiplex PCR to differentiate between Acinetobacter calcoaceticus and Acinetobacter genomic species 3. J Clin Microbiol 48 (12): $4592-$ 4594. DOI: $10.1128 / \mathrm{jcm} .01765-10$.

Huang JJ, Hu HY, Wu YH, Wei B, Lu Y. 2013. Effect of chlorination and ultraviolet disinfection on tetA-mediated tetracycline resistance of Escherichia coli. Chemosphere 90 (8): 2247-2253. DOI: 10.1016/j.chemosphere.2012.10.008.

Hsu LY, Apisarnthanarak A, Khan E, Suwantarat N, Ghafur A, Tambyah PA. 2017. Carbapenem-resistant Acinetobacter baumannii and Enterobacteriaceae in South and Southeast Asia. Clin Microbiol Rev 30 (1): 1-22. DOI: 10.1128/CMR.masthead.30-1.

Kalal BS, Chandran SP, Yoganand R, Nagaraj S. 2020. Molecular characterization of carbapenem-resistant Acinetobacter baumannii strains from a Tertiary Care Center in South India. Infect 24 (1): 27 34. DOI: 10.22354/in.v24i1.824.

Karuniawati A, Saharman YR, Lestari DC. 2013. Detection of carbapenemase encoding genes in Enterobacteriace, Pseudomonas aeruginosa, and Acinetobacter baumanii isolated from patients at Intensive Care Unit Cipto Mangunkusumo Hospital in 2011. Acta Med Indones-Indones J Intl Med 45 (2): 101-106.

Kateete DP, Nakanjako R, Namugenyi J, Erume J, Joloba ML, Najjuka CF. 2016. Carbapenem resistant Pseudomonas aeruginosa and Acinetobacter baumannii at Mulago Hospital in Kampala, Uganda (2007-2009). SpringerPlus 5: 1308. DOI: 10.1186/s40064-016-29867 .

Kuntaman K, Shigemura K, Osawa K, Kitagawa K, Sato K, Yamada N, Nishimoto K, Yamamichi F, Rahardjo D, Hadi U, Mertaniasih NM, Kinoshita S, Fujisawa M, Shirakawa T. 2018. Occurrence and characterization of carbapenem-resistant Gram-negative bacilli: A collaborative study of antibiotic-resistant bacteria between Indonesia and Japan. Intl J Urol 25 (11): 966-972. DOI: 10.1111/iju.13787.

Li S, Jia X, Li C, Zou H, Guo Y, Zhang L. 2018. Carbapenem-resistant and cephalosporin-susceptible Pseudomonas aeruginosa: A notable phenotype in patients with bacteremia. Infect Drug Resist 11: 12251235. DOI: $10.2147 /$ idr.s174876.

Lukovic B, Gajic I, Dimkic I, Kekic D, Zornic S, Pozder T, Radisavljevic S, Opavski N, Kojic M, Ranin L. 2020. The first nationwide multicenter study of Acinetobacter baumannii recovered in Serbia: Emergence of OXA-72, OXA-23 and NDM-1-producing isolates. Antimicrob Resist Infect Control 9: 101. DOI: 10.1186/s13756-02000769-8.

Marathe NP, Berglund F, Razavi M, Pal C, Dröge J, Samant S, Kristiansson E, Larsson J. 2019. Sewage effluent from an Indian Hospital harbors novel carbapenemases and integron-borne antibiotic resistance genes. Microbiome 7: 97. DOI: 10.1186/s40168-019-0710-

Miranda CC, de Filippis I, Pinto LH, Coelho-Souza T, Bianco K, Cacci LC, Picão RC, Clementino MM. 2015. Genotypic characteristics of multidrug-resistant Pseudomonas aeruginosa from hospital wastewater treatment plant in Rio De Janeiro, Brazil. J Appl Microbiol 115 (6): 1276-1286. DOI: 10.1111/jam.12792.

Moradali MF, Ghods S, Rehm BHA. 2017. Pseudomonas aeruginosa lifestyle: A paradigm for adaptation, survival, and persistence. Front Cell Infect Microbiol 7: 39. DOI: 10.3389/fcimb.2017.00039. 
Motbainor H, Bereded F, Mulu W. 2020. Multi-drug resistance of blood stream, urinary tract and surgical site nosocomial infections of Acinetobacter baumannii and Pseudomonas aeruginosa among patients hospitalized at Felegehiwot referral hospital, Northwest Ethiopia: A cross-sectional study. BMC Infect Dis 20: 92. DOI: 10.1186/s12879-020-4811-8.

Nishio H, Komatsu M, Shibata N, Shimakawa K, Sueyoshi N, Ura T, Satoh K, Toyokawa M, Nakamura T, Wada Y, Orita T, Kofuku T, Yamasaki K, Sakamoto M, Kinoshita S, Aihara M, Arakawa Y. 2004. Metallo- $\beta$-lactamase-producing Gram-negative bacilli: Laboratorybased surveillance in cooperation with 13 clinical laboratories in the Kinki Region of Japan. J Clin Microbiol 42 (11): 5256-5263. DOI: 10.1128/jcm.42.11.5256-5263.2004.

Nordmann P, Poirel L. 2002. Emerging carbapenemases in Gram-negative aerobes. Clin Microbiol Infect 8 (6): 321-331. DOI: 10.1046/j.14690691.2002.00401.x.

Pfeifer Y, Wilharm G, Zander E, Wichelhaus TA, Göttig S, Hunfeld KP, Seifert H, Witte W, Higgins PG. 2011. Molecular characterization of blaNDM-1 in an Acinetobacter baumannii strain isolated in Germany in 2007. J Antimicrob Chemoter 66 (9): 1998-2001. DOI: $10.1093 / \mathrm{jac} / \mathrm{dkr} 256$.

Poirel L, Postron A, Nordmann P. 2012. OXA-48-like carbapenemases: The phantom menace. J Antimicrob Chemother 67 (7): 1597-606. DOI: $10.1093 / \mathrm{jac} / \mathrm{dks} 121$.

Ranjbar R, Zayeri S, Afshar D, Farshad S. 2019. Detection of OXA beta lactamases among clinical isolates of Acinetobacter baumannii isolated from Tehran Hospitals, Iran. Open Microbiol J 13: 68-72. DOI: $10.2174 / 1874285801913010068$.

Rizzo L, Manaia C, Merlin C, Schwartz T, Dagot C, Ploy MC, Michael I, Fatta-Kassinos D. 2013. Urban wastewater treatment plants as hotspots for antibiotic resistant bacteria and genes spread into the environment: A review. Sci Total Environ 447: 345-360. DOI: 10.1016/j.scitotenv.2013.01.032

Shams S, Hashemi A, Esmkhani M, Kermani S, Shams E, Piccirillo A. 2018. Imipenem resistance in clinical Escherichia coli from Qom, Iran. BMC Res Notes 11: 314. DOI: 10.1186/s13104-018-3406-6.

Spindler A, Otton LM, Fuentefria DB, Corção G. 2012. Beta-lactams resistance and presence of class 1 integron in Pseudomonas spp. isolated from untreated hospital effluents in Brazil. Antonie Van Leeuwenhoek 102 (1): 73-81. DOI: 10.1007/s10482-012-9714-2.

Ssekatawa K, Byarugaba DK, Wampande E, Ejobi F. 2018. A systematic review: The current status of carbapenem resistance in East Africa. BMC Res Notes 11: 629. DOI: 10.1186/s13104-018-3738-2.

Suwantarat N, Carroll KC. 2016. Epidemiology and molecular characterization of multidrug-resistant Gram-negative bacteria in Southeast Asia. Antimicrob Resist Infect Control 5 (15): 1-8. DOI: 10.1186\%2Fs13756-016-0115-6.

Taee SR, Khansarinejad B, Abtahi H, Najafimosleh M, Ghaznavi-Rad E. 2014. Detection of algD, oprL and exoA genes by new specific primers as an efficient, rapid and accurate procedure for direct diagnosis of Pseudomonas aeruginosa strains in clinical samples. Jundishapur J Microbiol 7 (10): e13583. DOI: 10.5812/jjm.13583.

Tafreshi N, Babaeekhou L, Ghane M. 2019. Antibiotic resistance pattern of Acinetobacter baumannii from burns patients: Increase in prevalence of blaOXA-24-like and blaOXA-58-like genes. Iran J Microbiol 11 (6): 502-509.

Vrancianu CO, Gheorghe I, Czobor IB, Chifiriuc MC. 2020. Antibiotic resistance profiles, molecular mechanisms and innovative treatment strategies of Acinetobacter baumannii. Microorganisms 8 (6): 935. DOI: 10.3390/microorganisms8060935.

Wang TH, Leu YS, Wang NY, Liu CP, Yan TR. 2018. Prevalence of different carbapenemase genes among carbapenem-resistant Acinetobacter Baumannii in blood isolates in Taiwan. Antimicrob Resist Infect Control 7: 123. DOI: 10.1186/s13756-018-0410-5.

Woodford N, Ellington MJ, Coelho JM, Turton JF, Ward ME, Brown S, Amyes SGB, Livermore DM. 2006. Multiplex PCR for genes encoding prevalent OXA carbapenemases in Acinetobacter spp. Intl J $\begin{array}{lllll}\text { Antimicrob Agents } 27 & \text { (4): } & & \end{array}$ 10.1016/j.ijantimicag.2006.01.004.

Zhang L, Ma X, Luo L, Hu N, Duan J, Tang Z, Zhong R, Li Y. 2020a. The prevalence and characterization of extended-spectrum $\beta$ lactamase- and carbapenemase-producing bacteria from hospital sewage, treated effluents and receiving rivers. Intl J Environ Res Public Health 17 (4): 1183. DOI: 10.3390/ijerph17041183.

Zhang S, Huang J, Zhao Z. Cao Y, Li B. 2020b. Hospital wastewater as a reservoir for antibiotic resistance genes: a meta-analysis. Front Public Health 8: 574968. DOI: 10.3389/fpubh.2020.574968. 